\title{
Dynamics of inhomogeneous elastic half-space under moving load
}

\author{
Bakhtiyor Yuldashev ${ }^{1, *}$ and Sagdulla Abdukadirov ${ }^{2}$ \\ ${ }^{1}$ Department of Theoretical and Constructional Mechanics, Tashkent Institute of Irrigation and \\ Agricultural Mechanization Engineers, 39 KoriNiyoziy str., Tashkent 100000, Uzbekistan \\ ${ }^{2}$ Department of Building Mechanics and Seismic Resistance of Structures, Tashkent Institute of \\ Architecture and Construction, 13 Navoi str., Tashkent 100000, Uzbekistan
}

\begin{abstract}
Wave processes in an elastic half-space covered with an elastic layer and (or) a thin elastic plate are considered in the paper. External load moves along the free surface. In the stationary statement, the waveguide properties of the system are determined. The multiple roots of the dispersion equations are revealed and the critical load velocities, leading to the initiation of resonant processes, are determined. In the case when the load moves with the velocity of the Rayleigh wave, additional resonances determined by the structure can be realized in the structure under consideration. It is revealed that Rayleigh resonance exists for long waves only. Numerical solutions are obtained that make it possible to trace the development of resonant excitations. The models of simple structures that have dispersive properties in the medium wave zone are analyzed, such as a thin plate on an elastic base; a model with an attached inertial medium. Analytical solutions have been obtained for these models. Computer simulations conducted simultaneously allow us to analyze the quantitative features of process throughout the entire time period of the load effect. The numerical and asymptotic solutions are compared.
\end{abstract}

\section{Introduction}

It is known, the consideration of resonant waves in structurally inhomogeneous rigid bodies has been initiated in $[1,2]$, where a plane statement was considered, while the dispersion properties of such structures and the existence of critical velocities have been studied earlier in [3]. In [4], the same problem has been solved for the case of cylindrical symmetry.

Note that the study of resonance phenomena in the case of homogeneous bodies was carried out much earlier. First of all, let us point out the pioneer study [5], where the plane problem was solved for a free half-space and a surface load moving with the Rayleigh velocity. It was shown that, firstly, in this case the stationary limit of the solution is absent; secondly, the particle velocities and the stresses near the surface increase linearly. Then various aspects of resonant waves for plates and cylindrical shells interacting with acoustic media have been studied [6-10]. Special points on the dispersion curves in the case of a

\footnotetext{
* Corresponding author: bakhtiyor.yuldashev@bk.ru
} 
hollow cylinder were considered in [6-9], asymptotic and computer solutions of bending resonance waves were obtained in $[11,12]$ for various types of moving loads.

It is important to note that the main contribution to the theory of resonant waves in rigid bodies and structures belongs to L. I. Slepian (see, for example, [9, 10]). An effective analytical tool for studying the resonant processes turned out to be the method, developed by him, of inverting the Laplace-Fourier double images in the vicinity of a moving wave ( $x=c t, c$ is the wave velocity, $t$ is time). This approach allowed us to obtain asymptotic solutions of processes (for large values of time) in a closed form. On the basis of this method, a number of problems, including practical applications, have been investigated in $[3,4,6,9,11,12]$. Numerous aspects of problems with moving loads that do not consider the resonant waves can be found, for example, in [13-16, 19-21] (a detailed review of publications in [15] should be especially noted), but we will not discuss it here.

Despite the fact that processes with moving loads have been studied in many sources, the problem posed in the title of this paper, which has obvious theoretical and practical significance, requires further analysis. Until now, it was unclear what type of singular points on the dispersion curves and how many of them appear in the case of different structures, what common and distinctive features have the Rayleigh and medium wave resonance processes? Which of them is more dangerous from the point of view of the response of the construction under consideration?

These and other issues are addressed in this paper.

\section{Method}

Consider a dynamic problem for the plane system: thin plate - thick layer - halfspace. On the external surface $(y=0)$ beginning at time $t=0$, normal surface stresses of a given magnitude $Q$ move with constant velocity $V$ along the right and left directions of axes $x$.

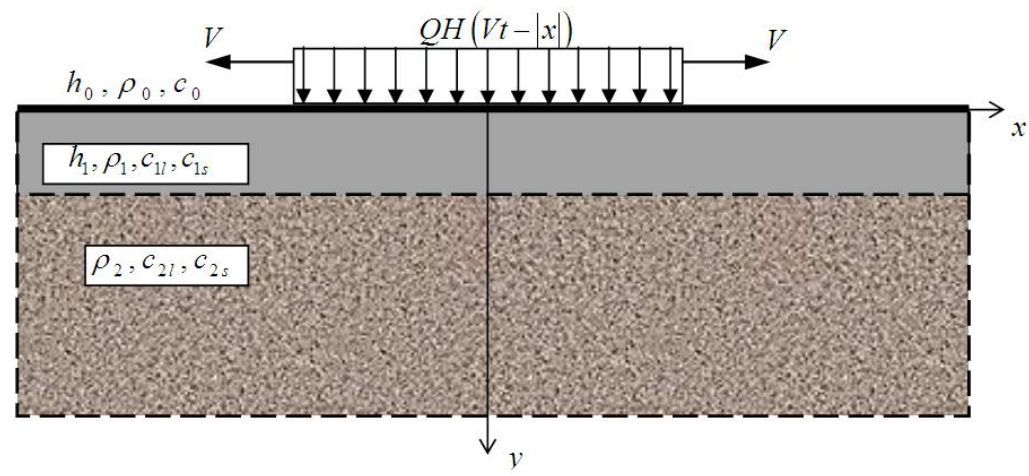

Fig. 1. The considered system: thin plate-layer-halfspace

In figure 1 , the system geometry is shown and the following significations are used: $H$ is the Heaviside step function, numbers $0,1,2$ refer to the plate, layer and halfspace, respectively; $c_{o}$ is the velocity of longitudinal waves in the plate, $\rho_{j} \quad(j=0,1,2)$ are densities, $h_{j} \quad(j=0,1)$ are thicknesses, subindices $l$ and $s$ related to velocities of longitudinal and shear waves, respectively: $c_{j l}$ and $c_{j l}(j=1,2)$.

The theory of dynamic elasticity describes the motion of the layer $\left.0<y<h_{1}: j=1\right)$ and halfspace $\left(y>h_{1}: j=2\right)$ as follows: 


$$
\begin{aligned}
& \ddot{u}=c_{j l}^{2} u_{j, x x}^{\prime \prime}+c_{j s}^{2} u_{j, y y}^{\prime \prime}+\left(c_{j l}^{2}-c_{j s}^{2}\right) w_{j, x y}^{\prime \prime}, \\
& \ddot{w}=c_{j l}^{2} w_{j, y y}^{\prime \prime}+c_{j s}^{2} w_{j, x x}^{\prime \prime}+\left(c_{j l}^{2}-c_{j s}^{2}\right) u_{j, x y}^{\prime \prime},
\end{aligned}
$$

while the classic Bernoulli equation is used for plate dynamic bending:

$$
y=0: \ddot{w}+c_{0}^{2}\left(h_{0}^{2} / 12\right) w_{x x x x}^{(j v)}=(P-R) / p_{0} h_{0},
$$

where $P=Q H(V t-|\mathrm{x}|)$ is a moving step load, $R$ is the normal reaction of the layer to the plate motion:

$$
R(x, t)=\sigma_{y y}^{(1)}(x, 0, t)=p_{1}\left\lfloor c_{1 l}^{2} w_{1, y}^{\prime}+\left(c_{1 l}^{2}-2 c_{1 s}^{2}\right) u_{1, x}^{\prime}\right\rfloor
$$

All the components of the composition are connected by a rigid contact excluding longitudinal connection between the plate and layer (or the halfspace in the reduced system: plate-halfspace) which are assumed to be absent. So the following relations are proved:

$$
\begin{gathered}
y=0: w_{1}(x, 0, t)=\sigma_{x y}^{(2)}=0 ; \\
y=h_{1}: u_{1}=u_{2}, w_{1}=w_{2}, \sigma_{y y}^{(1)}=\sigma_{y y}^{(2)}, \sigma_{x y}^{(1)}=\sigma_{x y}^{(2)} . \\
y \rightarrow \infty: u_{2} \rightarrow 0, w_{2} \rightarrow 0
\end{gathered}
$$

Note that the load source is manifested here somewhat conditionally, it is convenient for analytical and numerical procedures, there is no problem to use the sources appeared in practical problems. For example external pressure waves caused by the air blast in a far field; the action of a plane landing onto a strip; internal sources related to earthquake or underground explosions (then the superposition method of wave theory can be used to calculate the parameters of the moving surface loading).

In the steady-state formulation, the solution of the problem (1) - (4) is found as a superposition of Fourier harmonics, $\exp [i q(x \pm c t)-\varsigma y]$, propagating along the $x$-axis and exponentially decaying at $y \rightarrow \infty$. Factor $\varsigma$ is calculated from Fourier-transforms of original equations for the halfspace. Then, by using the boundary conditions, the dispersion equation connecting the phase velocity, $c$, and the wave number, $q$, is obtained. This equation is transcendental, it has a cumbersome structure, and its formal expression only is presented below:

$$
L\left(q, c ; h_{0}, c_{0}, h_{1}, c_{1 l}, c_{2 l} \cdot p_{1}, c_{2 l}, c_{2 s}, p_{2}\right)=0
$$

In the general case, the analytical solution of Eq. (5) it is not possible to obtain, but there is no problem its computer solving. The some complexity, however, is to conduct (more or less) analysis of its solutions $\mathbf{0}$ dependences $c=c\left(q ; \sum\right)$ where $\sum$ is the set of nine parameters signed in (5). Using some of them as measurement units, the set of free parameters can be significantly reduced. Below calculation results are presented in the way to describe common dispersion features with the minimal varying of parameters. 


\section{Results}

The aim of analysis of Eqn. (5) is to find special points and examine the behavior of dispersion curves in their vicinities.

\subsection{Thin plate-halfspace}

First, consider a system: thin plate-halfspace, which is the simplest (single-mode) special case of the considered system. Formally, to obtain the mathematical formulation of the dynamics of this reduced system, there is enough to equating parameters of the layer and the halfspace. We introduce the following notation: $c_{l}=c_{1 l}=c_{2 l}, c_{s}=c_{1 s}=c_{2 c}, \rho=\rho_{1}=\rho_{2}$. Then Eqn. (5) is written as follows:

$$
\rho_{0} q \sqrt{1-c^{2}}\left(c^{2}-c_{0}^{2} q^{2} / 12\right) c^{2}+c_{s}^{4} L_{R}=0, L_{R}=\left(2-c^{2} / c_{s}^{2}\right)^{2}-4 \sqrt{1-c^{2}} \sqrt{1-c^{2} / c_{s}^{2}}
$$

where $c_{1}, \rho$ and $h_{o}$ are taken as measurement units. Here $L_{R}=0$ is the Rayleigh equation for a free halfspace (its single real root is $c=c_{R}$ ), and. Eqn. (6) has a single mode $c=c(q)$, which is real if $c<c_{s}$.

If $q \rightarrow 0$, then $c \rightarrow c_{R}$ : a plate of finite rigidity and mass does not influence the long wave dispersion (more accurately: the infinitely long wave dispersion). The asymptotic behavior of phase velocity c obtained from (6) is

$$
\begin{aligned}
& c=c_{R}\left[1-\alpha \rho_{0} q+0\left(q^{2}\right)\right] \alpha=1 / 4 \sqrt{1-c_{R}^{2}}\left(c_{R} / c_{s}\right)^{2} L_{1}^{-1} \succ 0, \\
& L_{1}=\left(1+c_{s}^{2}-2 c_{R}^{2}\right)\left[\left(1-c_{R}^{2}\right)\left(1-c_{R}^{2} / c_{s}^{2}\right)\right]^{2}-2+c_{R}^{2} / c_{s}^{2}
\end{aligned}
$$

If $\mathrm{q}$ is relatively small, $\mathrm{c}$ linearly decreases with $\mathrm{q}$ increasing. The decreasing rate in $\mathrm{c}$ strongly depends on the plate mass and, vice versa, is independent of the plate rigidity. With further increase in $q$, the decay of $c(q)$ within a middle spectrum stops, the dispersion curve reaches minimum $\left(q_{m}=q_{*}, c_{m}=c_{*}\right)$, and after that it monotonically rises up to $c=c_{s}$ (remind that real values of c are only if $c \leq c_{s}$ ).
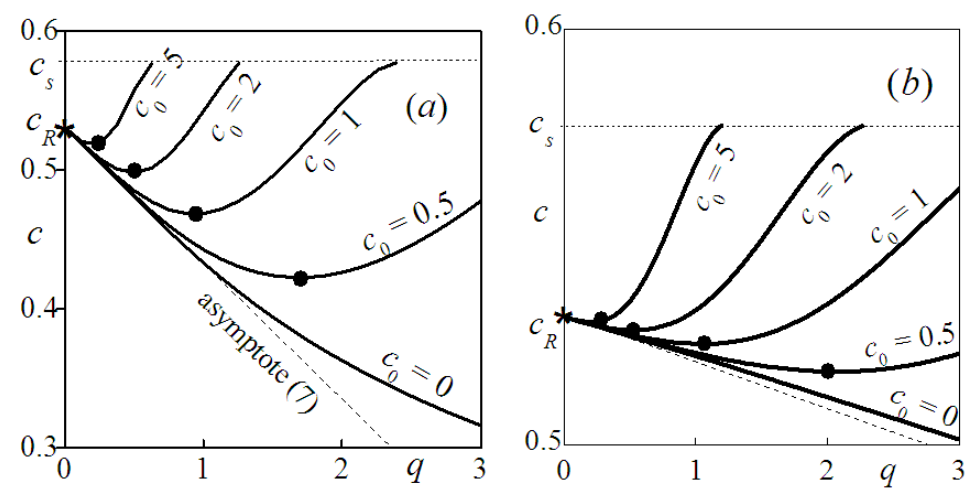

Fig.2. Dispersion curves for system: plate-halfspace, $(a)$ "heavy" plate, $c_{0}=2 ;(b)$ "light" plate, $c_{0}=$ 0.5 . 
Black circles in figure 2 are special points (minima) in the middle wave spectrum, while the asterisk is the special point of the Rayleigh surface resonance. Asymptote (7) coincides with a linear part of all curves. Note that here and in the subsequent examples, the Poisson ratios are the same: $v_{1}=v_{2}=0,25$, then $c_{k s}=c_{k l} / \sqrt{3} \approx 0.5774 \quad(k=1,2)$.

It can be shown that points of the minimum are inside the upper domains between two straight lines: asymptote (7) and the plate bending mode $c=c_{0} h q / \sqrt{12}$. Coordinates $q_{m}, c_{m}$ can be obtained from the following formulas:

$$
\left(p_{0} / c_{0}\right) c_{m}^{5} \sqrt{1-c_{m}^{2}}+\sqrt{3 / 8} L_{R}\left(c_{m}\right)=0, q_{m}=2 c_{m} / c_{0}
$$

So, in the considered case, two critical velocities exist: $V_{c r}=c_{R}$ in a long wave spectrum $(q \rightarrow 0)$ with a low frequency $(\omega \rightarrow 0)$, and $V_{c r}=c_{m}$, within a medium wave spectrum $\left(q=q_{m}\right)$.

If it is possible to approximate the dispersion curve in the vicinity $q_{m}$ by dependence $c \approx c_{m}+\beta\left(q-q_{m}\right)^{n}, \beta$ is constant, then the larger index $n$, the less the dispersion in the vicinity $q_{m}$, the wider becomes the wavelength spectrum that shapes the resonance disturbances and the more intense is their growth in time. As it is shown in [10], with the existing the above-mentioned approximation, the resonance growth rate is asymptotically proportional to $t^{(n-1) / n}(t \rightarrow \infty)$, while number $n(n \geq 2)$ is the first natural number for which $\partial^{n} c / \partial q^{n} \neq 0$. Therefore, in the case of a light and pliable plate (i. e. small $\rho_{0} a n d c_{0}$ ) resonance regimes in the medium wave spectrum are to be suspected as more intensive. On the other hand, with increase in $c_{0}$ the value of $c_{m}$ approaches $c_{R}$, while $q_{m}$ is removed into the long wave domain. This fact shows the possibility of a strong superposition of surface waves in the halfspace $\left(V_{c r}=c_{R}, q \rightarrow 0\right)$ and bending waves in the plate $\left(V_{c r}=c_{m}, q=q_{m}\right)$, which will considerably strengthen disturbances if $V$ is within interval $\left(c_{m}, c_{R}\right)$.

\subsection{The system thin plate-thick layer-halfspace}

Here as distinct from the previous case infinite number exist of the dispersion equation roots (modes) corresponding free wave modes propagating in a layer of finite thickness. If $q \rightarrow 0$, then as in the previous case $c \rightarrow c_{R 2}$, the Rayleigh velocity in the halfspace. The first (lower) mode has real roots, whereas the higher modes can be real or complex depending on the relation between system parameters. A set of special points can exist in these modes.

Longwave $(q \rightarrow 0)$ asymptote of the dispersion equation (5) is obtained as the following:

$$
L=c_{2 s}^{4} L_{R 2}+q\left[\rho_{0} c^{4} \sqrt{1-c^{2}}+\rho_{1} h_{1} \Phi\left(c_{1 l} ; c_{1 s}, c_{2 s}\right)\right]
$$


where $\Phi$ is a finite function. The measurement units are: $\rho_{2}, c_{2 l}$ and $h_{0}$. Remind, we assume $v_{0}=v_{1}=v_{2}=0,25$, then $c_{s} / c_{1}=0.577$ and $c_{R}=0.92$. Then only five free parameters are remain in the problem:। I $\rho_{0}, c_{0}, \rho_{1}, h_{1}, c_{1 l} l$.

As in the previous case, a linear asymptote $c(q)$ is proved by Eq. (9). Here the Rayleigh velocity is also critical for relatively long waves and the plate stiffness does not influence the longwave asymptote.

The main distinction that brings the layer existing is that velocity $c(q)$ can change drastically (decrease or increase) with growth in q. Obtaining analytical estimations of special points in the middle spectrum turns out problematic, but in this case, there is not problematic for the numerical solutions of the original dispersion equation shown as the formal expression in (5).

Below, in figure $3 \mathrm{a}, \mathrm{b}$ first modes are depicted calculated for a set of structure parameters, while four first modes can be observed in figure $3 \mathrm{c}$. For a relatively rigid and heavy layer $\left(\rho_{1}>\rho_{2}, c_{1 l}>c_{2 l}\right)$, the first mode can receive points of maximum and minimum in the medium wave spectrum: see examples in figures $4 \mathrm{a}, \mathrm{b}$.
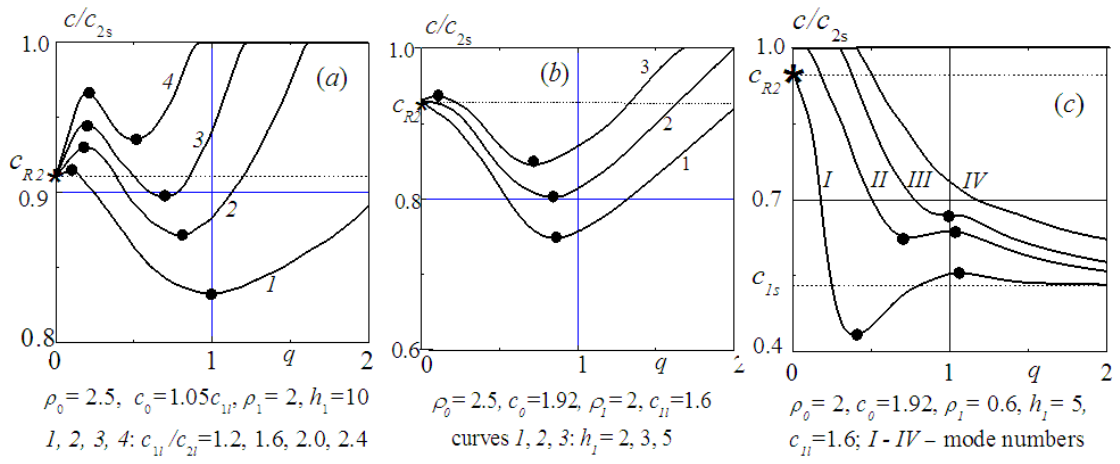

Fig. 3. Dispersion curves in the system: plate-layer-halfspace.

In the example for a lighter $\left(\rho_{1}=\rho_{2}\right)$ and more pliable layer $c_{1 l}<c_{2 l}$. I I Figure $\mathbf{3}, \mathbf{c}$, curves of the first and second modes have maximum and minimum, while the third mode they has an inflection point with a tangent parallel to the axis of $q$. The presence of diverse special points in the narrow spectrum of different modes testifies to the possibility superposing of different oscillation forms with close wavelengths in a narrow range of critical velocities.

\subsection{Non-steady-state problem. Development of resonant waves}

The diversity of special points in a wide spectrum revealed above proves the development of a set of resonant disturbances if the surface load moves with critical velocities VCR = $\mathrm{cm}$ and $\mathrm{VCR}=\mathrm{cR}$. Such processes were examined on the basis of direct numerical modeling of the problem considered. With this aim, an explicit finite-difference scheme is applied with using the special method of the mesh dispersion minimization initially introduced in [17] and developed in [18]. The method enables long- and short-wave components to be calculated with the same accuracy with a static difference mesh $[19,20$, 21].

In Figure 4, an initial stage of resonant wave formation in the system plate-halfspace is shown for two critical velocities of the moving step load: $V=c_{R}$ and $V=c_{m}$ and 
$V_{C R}=c_{R}$. The depicted curves are rising values of normalized normal reaction $\mathrm{R} / \mathrm{Q}$ in the mentioned cross-sections on the surface $(\mathrm{y}=0)$. Measurement units are $h_{0}, c_{0}, \rho_{0}$. One can see a clear distinction between these two cases: (a) a strong rise and a fixed frequency of the flexural resonance at $V=c_{m}$, while a weak rise and decreasing frequency are detected with time if $V=c_{R}$. Such peculiarities can partially be forecasted by the analysis of dispersion roots in vicinities of special points, where noticeable flattening of the dispersion curve in the vicinity of the minimum point $\left(q_{m}, c_{m}\right)$ is detected, which means to a decrease in the level of dispersion and, consequently, the increase in the growth rate of resonant perturbations. On the other hand, a relatively strong dispersion is revealed in the point corresponding to the Rayleigh resonance $\left(q=0, c=c_{R}\right)$. Such estimations can have a local character without claiming to be a kind of the generality: in the beginning of the wave process, it is problematically to establish the common characteristics of propagation of nonsteady-state perturbations. In the next Section, we have tried to build analytical solutions allowing highlighting the physical consequences of interest.
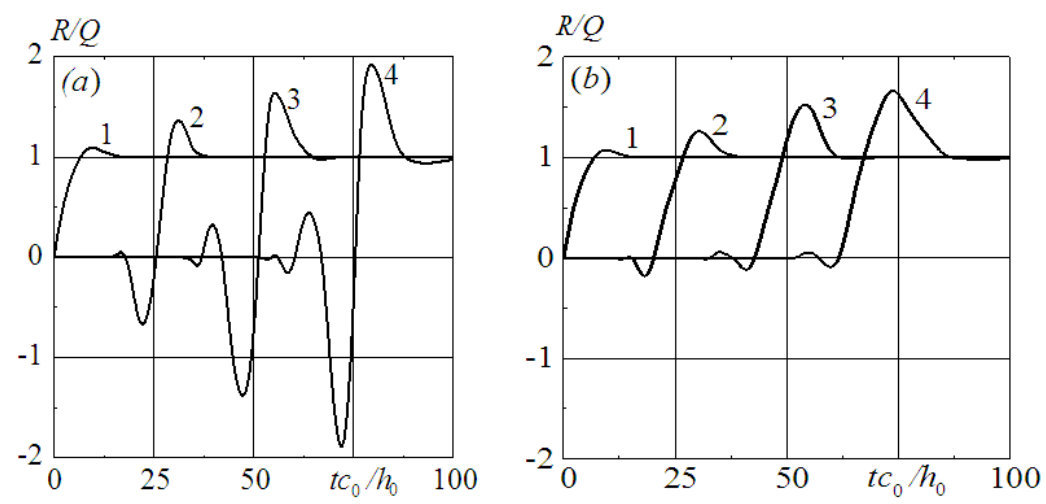

Fig. 4. Formation of resonant process in the system plate-halfspace: ${ }_{-2}^{-}=0.4, c_{2 l}=1$. Curves 1, 2, 3, 4 correspond to cross-sections $x=0,10,20,30 .(a): V=c_{m}=0.48,(b): V=c_{\mathrm{R}}=0.53$.

The similar process is detected in the system plate-layer-halfspace. In figure 5 the results are shown calculated in the case of a relatively rigid and heavy halfspace. It can be seen, that in the case of the bending resonance $(\mathrm{V}=\mathrm{cm})$, the growth of perturbations is more pronounced.
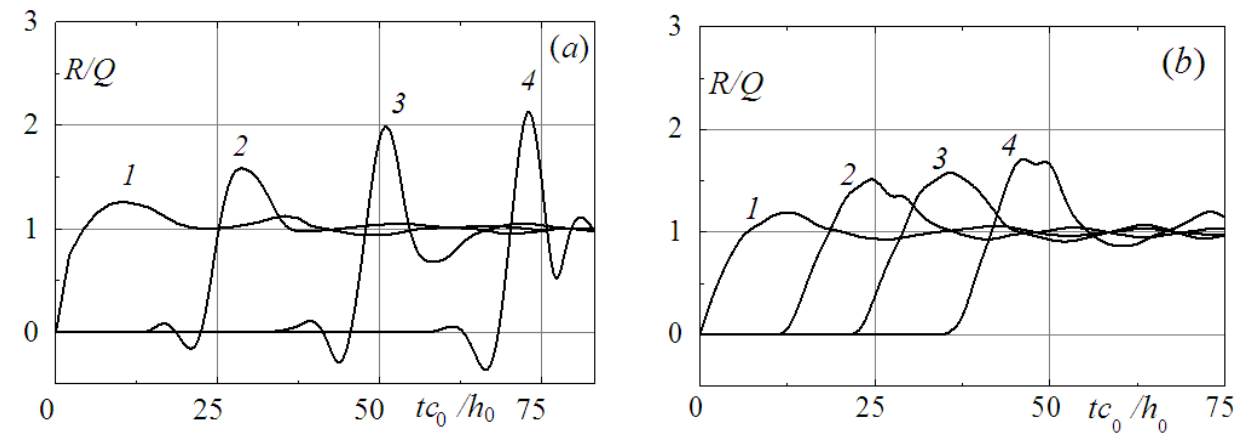

Fig.5. Formation of resonant process in the system plate-plate-halfspace: $h_{1}=$

5, $\rho_{1}=1.25, c_{11}=1, \rho 2=0.4, c 21=0.4$. Curves $1,2,3,4$ correspond to crosssections $X=0,10,20,30$. (a): $V=c_{m}=0.48,(b): V=c_{\mathrm{R}}=0.53$. 


\subsection{Simplified models}

Unfortunately, obtaining analytical solutions for resonant waves in the considered systems is problematical. However, such solutions can be successively found on the basis of the wellknown approach in which the original complex structure is changed by a simplified structure possessing the closest spectral properties in a detailed spectral band. This approach is notably applicable in the considered above case of flexural resonances where a narrow spectrum surrounding a special point is of interest. With this aim, we have used below two simple models: (a) thin plate upon an elastic foundation and (b) the same system supplemented with distributed inertial masses connected with the plate by inertionless elastic springs (it is clear that there are no Rayleigh points in these models).

The measurement units for these two models are parameters of the plate: $h, c, \rho$, other parameters of systems are indicated in figure 6 , the foundation rigidity $g$-model (a) (the single free parameter), and three free parameters in the model (b): $g ; G$ - the rigidity of links connected the plate with the amortized medium, and its mass $M$.

(a)

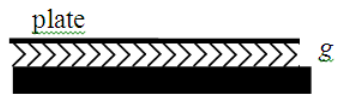

(b)

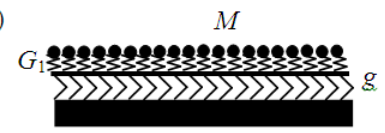

Fig. 6. Considered simplified systems.

The dimensionless equations describing dynamics of simplified models subjected to action of the travelling load are

$$
\begin{aligned}
& \text { (a) } \ddot{w}+(1 / 12) w_{x}^{(I V)}=P-R, R=g_{0} w ; \\
& \text { (b) } \ddot{w}+(1 / 12) w_{y}^{(I V)}=P-R, R=g w+G(w-W), \\
& M W-G(w-W)=o
\end{aligned}
$$

where $P=Q H(V t-|\mathrm{x}|)$ is the external travelling load, $R-$ is $(a)$ the elastic reaction of the foundation, $(b)$ the total reaction of the foundation and of the amortized medium.

Dispersion equations (5) for these models are disclosed as follows:

$$
\text { (a) } L(q, c, g)=q^{4} / 12-q^{2} c^{2}+g=0
$$

In the case of system $(a)$, a single special point - minimum is found:

$$
\text { (b) } L(q, c, g, G, M)=\left\lfloor q^{4} / 12-q^{2} c^{2}+g+G\right\rfloor\left[G-M q^{2} c^{2}\right\rfloor-G^{2}=0
$$

while in model (b), special numerical procedures to solving Eq. $(11, b)$ are required to find the coordinates of special points. As it was numerically obtained, the model (b) can have from one (minimum) to three (minimum, maximum and inflection) such points, depending on structure parameters.

By variations of free parameters in Eq. (11) we choose such of them that ensure the closest proximity of the dispersion curves of the original and the simplified model within a given spectral band. Such a procedure can be completed, for example, by the least square method. It is clear that the model $(b)$ has substantially more possibilities than the model (a) to coincide the dispersion patterns in the original model and simplified those. Nevertheless, we consider just the simplest model $(a)$ below with the aim to build an analytic solution for the explored problem. 
In figure 7 , dispersion curves are depicted calculated from $(11$, a) for some values of rigidity g. The special points of minimums can be indicated.

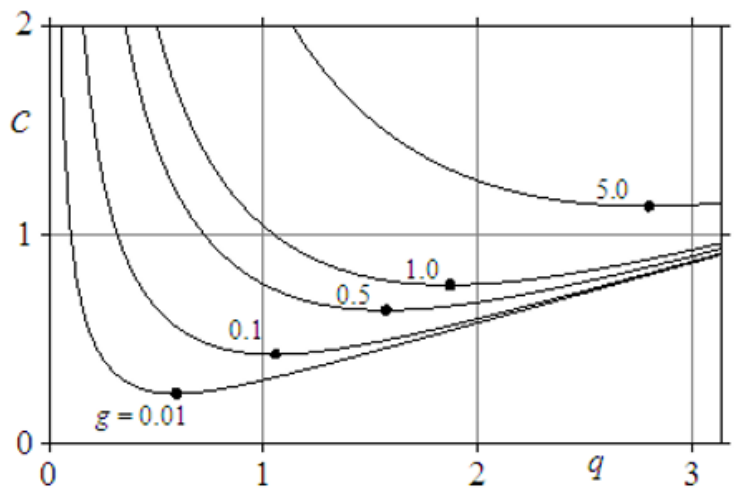

Fig.7. Dispersion curves for the model ( $a$ ).

\subsection{Asymptote of the flexural resonant process}

Asymptotic solutions we have built basing on the Slepyan approach [9], in which a double Laplace-Fourier transform is reduced to the Laplace transform at the ray $x=V_{c r} t$ with further asymptotic expression of the double Laplace-Fourier images in the vicinity $q=q_{m}$ (the wave number $q$ is the Fourier transform parameter) and for a great values of time: $t \rightarrow \infty$ (that correspond to asymptotic condition $s \rightarrow 0$ in the Laplace-Fourier images, where $s$ is the Laplace transform parameter). After these procedures, the precise reversion of the joint Laplace-Fourier transform is completed.

Below we omit rather cumbersome mathematical calculations and show the final formulas of the asymptotic solution to the problem $(10, a)$ :

$$
\begin{aligned}
& w(x, t)=\frac{\sqrt{t}}{\pi q_{m}^{2} c_{m}\left(1-c_{m}^{2}\right) \sqrt{\varphi}}\left[F_{1}(k) \cos \eta q_{m}+F_{2}(k) \sin \eta q_{m}\right] \\
& F_{1}(k)=\int_{0}^{\infty} \frac{\sin z^{2} \cos k z d z}{z^{2}}, \quad F_{2}(k)=\int_{0}^{\infty} \frac{\left(1-\cos z^{2}\right) \cos k z d z}{z^{2}} \\
& \varphi=\frac{1}{2}\left(q \frac{\partial^{2} c}{\partial q^{2}}\right)_{q=q_{m}} \neq 0, \quad k=\frac{\eta}{\sqrt{\varphi t}}, \quad \eta=c_{m} t-x
\end{aligned}
$$

Here $\eta$ is the stationary phase in the travelling wave. In the vicinity of this phase, wave pattern is marked by the growth of resonant perturbations proportional to $\sqrt{t}$; the oscillating process is described by the sum of envelopes $F_{1}$ and $F_{2}$ with the sinusoidal saturation with the carrier resonant frequency $\omega_{m}=c_{m} q_{m}$. Integrals $F_{1}$ and $F_{2}$ have the following analytical expressions: 


$$
\begin{aligned}
& F_{1}(k)=\sqrt{\frac{\pi}{2}}\left[\cos \left(\frac{k^{2}}{4}\right)+\sin \left(\frac{k^{2}}{4}\right)\right]-\frac{\pi|k|}{2}\left[C\left(\frac{k^{2}}{4}\right)-S\left(\frac{k^{2}}{4}\right)\right], \\
& F_{2}(k)=\sqrt{\frac{\pi}{2}}\left[\cos \left(\frac{k^{2}}{4}\right)-\sin \left(\frac{k^{2}}{4}\right)\right]-\frac{\pi|k|}{2}\left[1-C\left(\frac{k^{2}}{4}\right)-S\left(\frac{k^{2}}{4}\right)\right]
\end{aligned}
$$

where $\mathrm{C}(\ldots)$ and $\mathrm{S}(\ldots)$ are the Fresnel integrals. The graph expression of $F_{1}$ and $F_{2}$ can be seen in figure 8.
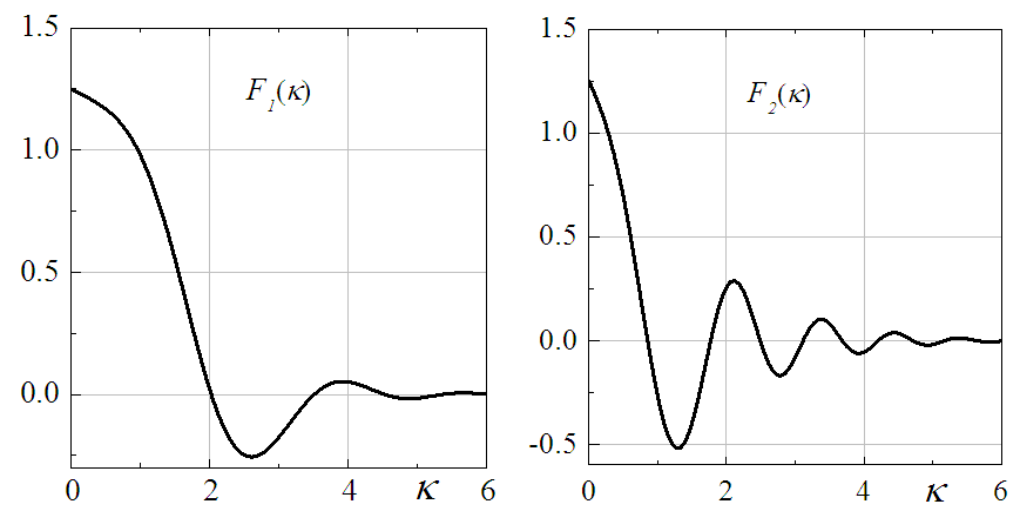

Fig.8. Envelopes of resonant waves.

\section{References}

1. S. Abdukadirov. Proc. of All-Union Conf: Applied Problems of Strength and Plasticity (Gorki University, Gorki, 1980)

2. S. Abdukadirov. Numerical Methods in Solid Mechanics, 11(6) (1980)

3. S. Abdukadirov, M. Stepanenko. J. Mining Science, 5 (1979)

4. S. Abdukadirov. J. Mining Science, 3 (1980)

5. R.V. Goldshtein. Appl. Math. Mech., 29(3) (1965)

6. L.I. Slepyan. The 6th All-Union Conf: Theory of Plates and Shells (Moscow, Nauka, 1966).

7. M.V. Ayzenberg, L.I. Slepyan. All-Union Symp. Transient Processes in Plates and Shells (Estonia, Tallinn, 1967)

8. M.V. Ayzenberg. Mechanics of Solids, 4(1) (1969).

9. L.I. Slepyan. Non-steady-state elastic waves (Leningrad, Sudostroenie, 1972)

10. L.I. Slepyan, O.V. Tsareva. Sov. Phys. Dokl., 32(6) (1987)

11. N.I. Alexandrova, I.A. Potashnikov, M.V. Stepanenko. J.Appl.Mech.Techn. Phys., 19(3) (1989)

12. M.V. Ayzenberg-Stepanenko. 25th Israel Conf. Mech. Engng., Technion (Israel, Haifa, 1994)

13. H. Ouyang, J.E. Mottershead. Numer. Meth. Eng., 72(10) (2007)

14. H.S. Zibdeh, I. Abu-Alshaikh. Int. J. of Vehicle Noise and Vibr., 4 (2008)

15. H. Ouyang. Mechanical Systems and Signal Processing, 25(6) (2011) 
16. P. Naga, M.R. Eatherton. Earthquake Engng \& Struct. Dyn., 43(5) (2014)

17. S.A. Abdukadirov, N.I. Pinchukova, M.V. Stepanenko. J. Mining Sci., 6 (1984)

18. S. Abdukadirov. 10th Int. Conf. on Materials Technologies and Modeling (MMT, 2018)

19. M.M. Mirsaidov, T.Z. Sultanov. Soil Mechanics and Foundation Engineering, 49(6) (2013)

20. M.M. Mirsaidov, T.Z. Sultanov, D.F. Rumi. Magazine of Civil Engineering, 39(4) (2013)

21. T.Z. Sultanov, D.A. Khodzhaev, M.M. Mirsaidov. Magazine of Civil Engineering, 45(1) (2014) 